\title{
Factors influencing the confidence and knowledge of nurses prescribing antiretroviral treatment in a rural and urban district in the Western Cape province
}

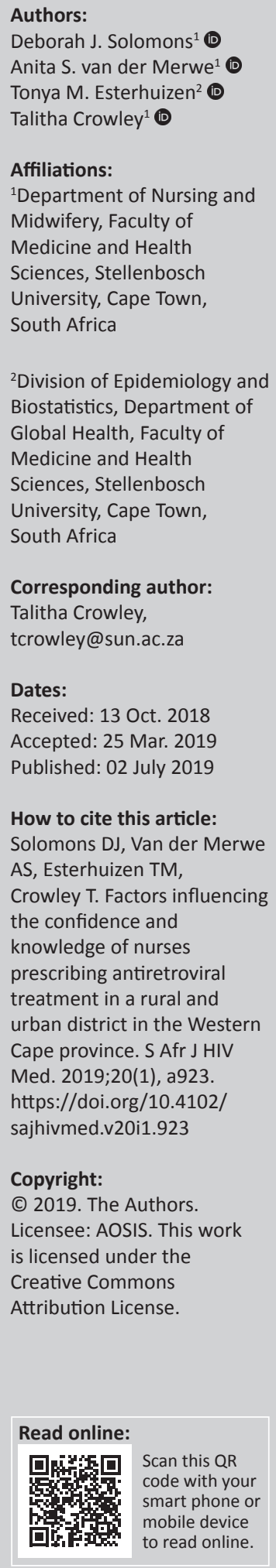

Corresponding author:

Talitha Crowley,

tcrowley@sun.ac.za

Dates:

Received: 13 Oct. 2018

Accepted: 25 Mar. 2019

Published: 02 July 2019

How to cite this article: Solomons DJ, Van der Merwe AS, Esterhuizen TM, Crowley T. Factors influencing the confidence and knowledge of nurses prescribing antiretroviral treatment in a rural and urban district in the Western Cape province. S Afr J HIV Med. 2019;20(1), a923. https://doi.org/10.4102/ sajhivmed.v20i1.923

\section{Copyright:}

C 2019. The Authors. Licensee: AOSIS. This work is licensed under the Creative Commons Attribution License.

Read online:

Background: Since the introduction of nurse-initiated and managed antiretroviral treatment (NIMART) in South Africa in 2010, initiation of antiretroviral therapy (ART) in primary care has become the responsibility of nurses. The continued success of this approach is dependent on factors such as adequate training and effective support systems.

Objectives: This study aimed to investigate factors influencing the knowledge and confidence of professional nurses in managing patients living with human immunodeficiency virus (HIV) in primary healthcare settings in a rural and urban district in the Western Cape.

Methods: A cross-sectional survey was conducted amongst 77 NIMART-trained nurses from 29 healthcare facilities to measure demographic details, influencing factors, HIV management confidence and HIV management knowledge.

Results: The majority of participants had adequate HIV management knowledge and reported being very confident or expert in the HIV management skills or competencies. Participants trained recently on local guidelines (Practical Approach to Care Kit) (3 years ago or less) had significantly higher knowledge scores. Regular feedback about clinic and personal performance was associated with higher HIV management knowledge. Participants who received NIMART mentoring over a period of 2 weeks had a higher mean confidence score compared to other periods of mentoring. A higher caseload of patients living with HIV was also associated with higher knowledge and confidence.

Conclusion: Training, mentorship and clinical practice experience are associated with knowledge and confidence. Recommendations include the strengthening of current training and mentoring and ensuring that NIMART-trained nurses are provided with regular updates and sufficient opportunities for clinical practice.

Keywords: HIV; NIMART; Primary Healthcare; Clinics; Nurses.

\section{Introduction and background}

South Africa has the largest antiretroviral treatment programme in the world. ${ }^{1}$ Antiretroviral treatment guidelines are continuously revised, consequently increasing the threshold for antiretroviral therapy (ART) treatment. Because of persistent human resource constraints in South Africa, task shifting from doctors to nurses to prescribe ART became essential to ensure that more patients living with human immunodeficiency virus (HIV) are initiated on lifesaving ART. ${ }^{2}$ Since the introduction of nurse-initiated and managed antiretroviral therapy (NIMART) in South Africa in 2010, there has been an increased demand for the training of professional nurses in HIV management in the primary healthcare (PHC) setting. The availability of NIMART-trained nurses in PHC clinics has improved the access of patients to HIV treatment. ${ }^{3}$

Although the shifting of tasks is a timely solution for human resource constraints, the continued success of the approach depends on factors such as adequate training and effective support systems. ${ }^{4}$ The World Health Organization (WHO) recommends that countries should adopt a methodical approach to coordinated, consistent and competencybased education that is needs-driven and approved. This will ensure that all healthcare workers are equipped with the appropriate competencies to undertake the tasks that they perform. ${ }^{5}$ 
Competency has been described as the knowledge, perceptions, skills, attitudes and standards that an individual develops or acquires through education, training and work experience, which can be used to depict particular occupational roles or functions against which personal performance may be assessed. ${ }^{6}$ Although it is ideal that all health professionals should be competent to undertake the tasks they perform, competency may be difficult to assess. The assessment of competency in the form of subjective, multiple-choice and standardised patient assessments may underemphasise significant domains of professional capability such as the integration of knowledge and skills, the framework of care, cooperation and patient-provider associations. ${ }^{7}$ It is even more challenging to assess the competency of clinicians in practice. In this study, selfassessment was used to measure how confident nurses are in performing HIV management skills. Knowledge questions were used to provide an objective assessment. Self-assessment is often used to help practising clinicians to identify their own strengths and weaknesses for continuous professional development. However, the process of assessing oneself is complex and never completely objective. Self-assessment can therefore not be used as an accurate measure of competency, but it can be used to help individuals identify gaps in their clinical performance. ${ }^{8}$ One such study conducted in the Eastern Cape province of South Africa found satisfactory self-efficacy in the clinical performance of NIMART amongst 358 trained nurses, with some limitations in their abilities regarding clinical evaluation. ${ }^{9}$ Care provided by providers with low levels of self-rated expertise who treat low numbers of HIV and AIDS patients tends to lead to less favourable patient outcomes. ${ }^{10}$

Factors such as training, mentoring and clinical experience have been found to influence the competency of healthcare providers. A systematic review revealed better clinical outcomes for patients treated by a provider with more training in HIV and AIDS care. ${ }^{10}$ There is very little research available on the evaluation of the different NIMART training courses and training outcomes. One study found that $62 \%$ of nurses who had been trained in NIMART were initiating patients on ART in the clinics where they were working, yet some of these nurses did not pass the open book exam after the training. ${ }^{11}$ In KwaZulu-Natal province, knowledge scores of nurses increased significantly after NIMART training, with a median post-test knowledge score of $77 \%$. However, the majority of nurses were not confident enough to practise their skills, which emphasised the need for continuous mentorship. ${ }^{12}$ A study in Khayelitsha, South Africa, showed an increase in the confidence of nurses to manage patients on ART after mentorship. ${ }^{13}$ Similarly, a study evaluating 5 years of NIMART mentorship in South Africa identified improved knowledge, attitudes and confidence perceived by nurses who received NIMART mentoring, but highlighted the need for mentoring to continue in light of continuous changes to treatment guidelines. ${ }^{14}$ In addition to mentoring, provider experience in HIV and AIDS care has shown to improve the quality of care. ${ }^{10}$
Although there is substantial evidence that nurses can provide high-quality treatment and care, several challenges have been identified, such as shortages of essential drugs, salary concerns, excessive workload, lack of practice standards, access to mentoring and infrastructural barriers.,11,15,16 This therefore highlights the need for continuous quality assurance in settings where NIMART is being implemented. ${ }^{17,18}$

No published studies could be found that specifically investigated the factors that influence the knowledge and confidence of nurses currently prescribing ART. Evaluating the HIV management confidence and knowledge of professional nurses who prescribe ART may help to improve ongoing NIMART training interventions. This study therefore aimed to determine the factors that influence the HIV management confidence and knowledge of professional nurses prescribing ART in a rural and urban district in the Western Cape province.

\section{Methods \\ Research design and setting}

A quantitative cross-sectional and analytical research design was used because NIMART training has been operational for several years and is in its implementation phase. ${ }^{19}$ The study was conducted in one urban and one rural district the City of Cape Town (City Health) and the Cape Winelands districts.

\section{Population and sample}

Based on a list obtained from the Department of Health, there were 256 nurses who were authorised to prescribe NIMART in the two districts. Of the five subdistricts in the Cape Winelands, three districts gave permission for the research and two declined. The assessable population, determined by contacting facilities, was 146 (67 in the City of Cape Town and 79 in the Cape Winelands). All the nurses authorised to prescribe NIMART for a period of 1 year were invited to participate in order to account for the clustering effect in the subdistricts. In the Cape Winelands, 49 (69\%) participants completed the questionnaires, 18 (25\%) refused to participate and four $(6 \%)$ were absent, on leave or not available when the research was conducted. In the City of Cape Town, 28 (42\%) participants completed the questionnaires, $22(33 \%)$ refused to participate and $17(25 \%)$ were absent, on leave or not available when the research was conducted.

\section{Instrumentation}

A self-completion questionnaire was used that was designed by the researcher based on the literature and previous instruments. The questionnaire measured demographic details, influencing factors, HIV management confidence and HIV management knowledge. The questionnaire was available in English only. In addition, facility statistics related to caseload were collected. 


\section{Pilot test}

A pilot test was conducted in the Stellenbosch sub-district. Participants in the sub-district were randomly selected to complete the questionnaire. Eight participants completed the questionnaire. After completion of the questionnaire, a few changes were made to the questionnaire. The pilot test data were not included in the main study.

\section{Validity and reliability}

Reliability and content validity of the instrument were ensured by making use of the literature, a review of the instrument by experts in the field of HIV and the pilot test. Content validity was determined by calculating a content validity index (CVI) for each item (called the I-CVI) in the questionnaire based on the feedback of five experts. The I-CVI was calculated by determining the percentage of experts who rated the specific item as relevant. For an item to be considered relevant, four of the five experts had to rate the item as relevant (an I-CVI score of 0.8 ). ${ }^{20}$ For six items in the questionnaire, one of the five experts rated the item as not relevant (an I-CVI score of 0.8). However, this is still acceptable according to the literature. For the remaining items, all the experts rated the items as relevant (an I-CVI score of 1). They made suggestions to improve the clarity of items and these suggestions were incorporated in the final instrument.

The Cronbach's alpha coefficient was used to establish the reliability of the Likert scale items that measured confidence. The previously reported Cronbach's alpha for the confidence items was $0.94 .{ }^{21}$ In this study, the Cronbach's alpha for the 22 confidence items was 0.95 .

\section{Data analysis}

Data were entered into Microsoft Excel by the researcher, imported and analysed using the Statistical Package for Social Sciences (SPSS) software. ${ }^{22}$ HIV management confidence and knowledge were measured as continuous variables by calculating the total scores. The scores were converted to percentages to facilitate interpretation. A higher score indicated more confidence. Descriptive statistics were used to describe the data and appropriate statistical tests were used to test for relationships between variables. As the confidence and knowledge scores were normally distributed, the student $t$-test was used to compare mean scores of two independent groups or categories of influencing factors and analysis of variance (ANOVA) for comparing more than two groups. The Spearman rho correlation coefficient was used to test for associations between continuous variables, for example, scores and the patient caseload of the participant (as the caseload was not normally distributed). A level of significance of $<0.05$ was used in this study.

\section{Ethical consideration}

Ethics approval to conduct the study was obtained from the Health Research Ethics Committee at Stellenbosch University (S14/12/268). Permission was further obtained from the Department of Health, City of Cape Town (City Health),
Western Cape Province and the appropriate Medical Superintendents from the chosen subdistricts. This study adhered to the ethical principles of the Declaration of Helsinki 2013. The researcher has the ethical responsibility to protect the human rights of the participants, such as their rights to privacy, confidentiality, autonomy, anonymity, fair treatment and protection from discomfort and harm.

\section{Results}

\section{Biographical data}

Most of the participants were recruited from healthcare facilities $(n=20,69 \%)$ in the Cape Winelands. The median headcount in facilities was 2496.5 (interquartile range [IQR] 1460.5-5441.5), indicating a high patient load in the facilities with relatively high variability between facilities. The mean age of participants was 43.6 years, with a standard deviation (s.d.) of 9.98 years, the youngest being 25 years of age and the oldest being 64 years of age.

Table 1 presents the categorical biographical data of the participants. Most of the respondents performed clinical work as a professional nurse. Participants in the 'other' category were providing different services, for example, the prevention of mother-to-child transmission (PMTCT) manager from head office prescribed ART if there was understaffing in certain clinics. Another participant was appointed as a psychiatric nurse but was also NIMART-trained and provided ART. One participant indicated that she was a clinical mentor but was also allocated to clinics to provide ART.

\section{Influencing factors}

The factors influencing confidence and knowledge included: (1) HIV management experience, (2) training, (3) continuous mentoring and support, (4) workload, motivation, facility equipment and general satisfaction, and (5) quality assurance mechanisms (see Table 2).

\begin{tabular}{lcc} 
TABLE 1: Biographical data of the participants. & & \\
\hline Variable & Frequency $(\boldsymbol{n})$ & $\%$ \\
\hline Gender & 71 & 7.8 \\
Male & & \\
Female & 44.2 & 57.1 \\
Highest professional qualification & 21 & 27.3 \\
Postgraduate diploma in primary healthcare & 8 & 10.4 \\
Diploma & 4 & 5.2 \\
Degree & & \\
Other & 63 & 81.8 \\
Current function & 8 & 10.4 \\
Clinical work as a professional nurse & 5 & 6.5 \\
Facility manager & 1 & 1.3 \\
Other & & \\
Clinical mentor & & \\
Type of facility & 41 & 53.2 \\
PHC clinic & 30 & 39.0 \\
Community day centre & 4 & 5.2 \\
Community health centre & 1 & 1.3 \\
Mobile clinic & 1 & 1.3 \\
Other & &
\end{tabular}

PHC, primary healthcare 
TABLE 2: Influencing factors.

\section{Influencing factor}

Frequency $(n)$

Confidence

Knowledge

\section{Experience}

Years of experience in HIV management

Less than 1 year

1 to less than 2 years

2-5 years

More than 5 years

Years of experience initiating ART

Less than 1 year

1 year to $<2$ years

2-5 years

More than 5 years

2. Training

Training course

Time of HIV management training (various courses)

$>3$ years

3 years or less

Time of PACK training

$>3$ years

3 years or less

Time of dispensing course

$>3$ years

3 years or less

None

NIMART training and mentoring

Time of NIMART training

$>3$ years

3 years or less

Duration of NIMART mentoring

1 week

2 weeks

$>2$ weeks -2 months

$>2$ months

Other

$p$

3. Continuous mentoring and support

How often does the ART doctor visit the clinic?

Daily

Daily

Monthly

Annually

Never

Are clinical mentors or a supervising clinician for HIV/TB/ART assigned to your clinic or district?

How often do you have contact sessions with your clinical mentor or a supervising clinician?

Daily

Weekly

Monthly

Annually

4. Workload, motivation, facility equipment and general satisfaction

Do you feel your workload is acceptable?

Yes

No

Do you feel motivated towards your work?

No

Do you feel that the facilities and equipment at the clinic are adequate for the delivery of HIV care?

Are you satisfied with your work conditions (e.g. work environment, salary and work hours)?

Yes

5. Quality assurance mechanisms

Feedback is received about

Personal performance relating to prescribing and monitoring patients on ART

Yes

Performance of the clinic related to the provision of ART

Yes

$\begin{array}{ll}46 & 59.7 \\ 31 & 40.3 \\ & \\ 47 & 61.0 \\ 30 & 39.0 \\ & \\ 32 & 41.5 \\ 12 & 15.5 \\ 33 & 43 .\end{array}$

5.2

0.25

0.45

28

6

34

6

31.2
68.8

$<0.0$

0.09

26
6.5
37.7

37.7
27.2

2.6

26

8.1

0.08

0.16

37.8

$\begin{array}{ll}9.7 & 0.06\end{array}$

0.75

0.75

3.0

.

$\begin{array}{cccc}40 & 51.9 & 0.78 & 0.06 \\ 28 & 36.5 & & \\ 1 & 1.3 & & \\ 0 & 0 & & \\ 8 & 10.4 & & \\ & & & \\ 57 & 74.0 & 0.49 & \\ 20 & 26.0 & & \\ & & & \\ 20 & 35.1 & 0.37 & \\ 19 & 33.3 & & \\ 14 & 24.6 & & \\ 3 & 5.3 & & \\ 1 & 1.8 & & \end{array}$

\begin{tabular}{cccc}
43 & 55.8 & 0.65 & 0.28 \\
34 & 44.2 & & \\
& & & \\
68 & 88.3 & 0.45 & 0.34 \\
9 & 11.7 & & \\
& & & \\
58 & 75.3 & 0.70 & \\
19 & 24.7 & & \\
& & & \\
40 & 51.9 & 0.27 & \\
37 & 48.1 & & \\
& & & \\
& & & \\
& & & \\
51 & 66.2 & 0.47 & \\
26 & 33.8 & & 0.01 \\
& & & \\
62 & 80.5 & 0.92 & \\
15 & 19.5 & & \\
\hline
\end{tabular}

therap

Note: The median number of monthly ART initiations by participants in the 3 months prior to the study was 7 (IQR 9) and the median number of ART follow-ups was 126.5 (IQR 347 ). 


\section{Human immunodeficiency virus management confidence}

The mean HIV management confidence score was $68.7 \%$ (95\% CI 66.3-71.1), with a minimum score of $45 \%$ and a maximum of $85 \%$. The participants reported the highest confidence in the use of ART stationery (with 51.9\% considering themselves experts) and performing a physical examination (with $50.6 \%$ considering themselves experts). They were less confident in identifying drug interactions in commonly used medications, because only $14.3 \%$ considered themselves to be experts, and stopping or switching drug treatments (with only 15.5\% considering themselves as experts). Low confidence was reported in prescribing for concurrent illnesses and in identifying the signs and symptoms of immune reconstitution inflammatory syndrome (fewer than $20 \%$ of participants considered themselves experts).

The distribution of the HIV management confidence score was the same across categories of district and facility type. Bivariate analysis indicated a low to moderate positive correlation between the HIV management confidence score and the average number of patients on ART followed up in the last 3 months $(r=0.328, p=0.004)$. The distribution of HIV management confidence scores was not the same across categories of how long NIMART mentoring lasted $(F[\mathrm{df} 4]=$ 4.6; $p=0.002$ ). Post hoc analysis revealed that participants who received NIMART mentoring for 2 weeks had significantly higher confidence scores than those who received mentoring for more than 2 months. None of the other influencing factors has significant associations with HIV management confidence (see Table 2).

\section{Human immunodeficiency virus management knowledge}

The mean HIV management knowledge score was $72.7 \%$ (95\% CI 69.8-75.6). The minimum score was $38 \%$ and the maximum $100 \%$. Participants had high knowledge scores regarding treatment for peripheral neuropathy $(97.4 \%$ indicated the correct answer) and the side effects of tenofovir, with $89.6 \%$ indicating the correct answer. High knowledge scores were also identified for identifying pneumocystis pneumonia (88.3\% correct) and ART contraindications $(88.3 \%$ correct). The participants were less knowledgeable regarding the treatment of toxoplasmosis $(22.1 \%$ correct $)$, oral hairy leukoplakia (39\% correct) and tinea capitis ( $41.6 \%$ correct). A few participants $(41.6 \%)$ demonstrated an understanding of virological failure and only $51.9 \%$ provided the correct answer for the question related to drug-drug interactions.

There was a significant difference in distribution of the HIV management knowledge score across categories of district $(t[\mathrm{df75}]=-2.9, p=0.004)$. Participants in the Cape Winelands had a lower mean knowledge score $(69.6 \%)$ compared to the mean knowledge score of participants in the City of Cape Town (78.2\%). Participants trained in Practical Approach to Care Kit (PACK - flowchart-based guidelines designed for assisting nurses manage various conditions in a primary care setting $)^{4} 3$ years ago or less had significantly higher knowledge scores $(t[\mathrm{df70}]=-3.5, p=0.001)$. Those with no training in dispensing had significantly higher knowledge scores, which is an unexpected finding. Knowledge scores of participants who indicated that they received regular feedback about their personal performance $(t$ df75] $=2.45$, $p=0.016)$ and the performance of the clinic related to the provision of ART $(t[\mathrm{df75}]=3.5, p=0.001)$ were significantly higher compared to those who did not. Bivariate analysis indicated a low to moderate positive correlation between the HIV management knowledge score and the average number of patients initiated on ART in the last 3 months $(r=0.357$, $p=0.002$ ) and a moderate positive correlation for the average number of patients on ART followed up $(r=0.386, p=0.001)$. A significant negative correlation was found between the HIV knowledge score and the average number of other or non-ART patients the participants managed in the past 3 months $(r=-0.367, p=0.001)$.

\section{Discussion}

Appropriate training is the beginning of the pathway to expertise. $^{5,23}$ In this study, a variety of HIV management training courses were attended by the participants. All of the participants were trained in PACK, completed an HIV management course and participated in NIMART training as per the NIMART guidelines of the Western Cape. ${ }^{13}$ Only $57.1 \%$ of participants had completed a dispensing course, although this did not affect confidence and was associated with lower knowledge scores. A dispensing certificate is not a requirement for NIMART in the Western Cape and most clinics have either a pharmacy assistant or a pharmacist who can dispense medication. Cameron et al. ${ }^{11}$ found that $79 \%$ of the nurse participants in their study had previous formal training in HIV management and 55\% had formal training in $\mathrm{PHC}$, which is comparable to the $57.1 \%$ of participants in the present study who had completed a postgraduate diploma in PHC. A qualification in PHC (Health Assessment, Treatment and Care [R48]) and a dispensing certificate are therefore not requirements to prescribe $\mathrm{ART}$ in the study context, but the PACK training and completing an HIV management course are. Although no cause and effect can be inferred, it can be deduced from the results that recent PACK training (3 years or less) is likely to improve the HIV management knowledge of nurses.

Clinical mentoring is depicted alongside clinical practise and continuous assessment on the pathway to competency and proficiency. ${ }^{22}$ The purpose of mentoring is to acquire skills to competently initiate, but also manage patients according to established clinical protocols. ${ }^{17}$ The Western Cape Department of Health guideline advises a minimum of $40 \mathrm{~h}$ of NIMART one-on-one mentorship following didactic training. ${ }^{13}$ In a study in the health districts of Tshwane (Gauteng Province), Nkangala (Mpumalanga Province) and Capricorn and Vhembe (Limpopo Province), the median mentoring period was 25 months. ${ }^{14}$ It therefore appears that there is no set period for mentoring. A total of 
80 cases are required to be seen by a nurse in consultation with a mentor, according to the Clinical Mentorship Guideline for Integrated Services, in order for the nurse to be authorised in NIMART. ${ }^{22}$ In the present study, all nurses had received NIMART mentoring. The findings from this study support a 2-week NIMART mentoring period. It may be that a 2 -week mentoring period is more intense. Perhaps those who were mentored for a longer period, for example, for more than 2 months, did not have intensive contact sessions and case studies or the contact sessions may have been too far apart. A study conducted by Orner et al. ${ }^{16}$ found that nurses and doctors were too busy for mentoring, which may result in less frequent contact sessions. A longer period of mentoring may therefore not translate to the acquisition of more knowledge and confidence in practice. This, however, needs to be explored further. Having an assigned mentor or the frequency of contact sessions with the mentor were not associated with the participants' level of confidence or knowledge. However, other studies have found mentoring to improve nurses' confidence, improving institutional barriers and the quality of patient care. ${ }^{13,14}$

Although mentoring was not associated with the participants' knowledge in the present study, participants who received regular feedback about their personal and clinical performance (classified in this study as part of quality assurance) had significantly higher knowledge scores $(75.2 \%)$ compared to those with limited or no feedback (67.9\%). Feedback was mostly received from the clinic manager or HIV and AIDS, STIs and TB coordinator. Regular feedback provided about individual and clinic performance related to the provision of ART is likely to influence nurses' HIV management confidence. ${ }^{4}$ However, the Clinical Mentorship Manual for Integrated Services ${ }^{22}$ distinguishes between clinical mentorship and supportive supervision. Although both have similar goals and some overlapping activities, supervision tends to emphasise health facility management and is more hierarchical, whereas mentoring is more focused on the enhancement of the skills of the mentee. Mentoring should therefore be more effective in improving the confidence and knowledge of nurses than feedback from supervisors. The above mentioned results may mean that mentoring is not currently being implemented or practised effectively.

Knowledge and confidence of the clinicians should increase as they gain experience..$^{22}$ In this study, the HIV management confidence and knowledge scores were the same across all categories of experience. However, the average number of patients living with HIV seen or the caseload may be an indication of the intensity of experience and was associated with both HIV management confidence and knowledge. It therefore appears that the total years of experience does not influence confidence and knowledge, rather it is more clinical practice experience, for example, the patients living with HIV caseload, that influences confidence and knowledge. None of the other health system influencing factors were associated with HIV management confidence and knowledge.
These may nonetheless still have an impact on overall service delivery and quality of patient care.

Recommendations based on the study findings include regular guideline updates to reinforce HIV management knowledge. Methods to strengthen mentoring should be engaged such as dedicated roving mentors or access to telephonic consultations. ${ }^{14}$ Dedicated mentoring may be costly and therefore non-governmental organisation support may be required to ensure mentor capacity. ${ }^{13}$ NIMART-trained nurses should be offered sufficient opportunities to practise their skills. As not all nurses may be exposed to a high caseload because of the PHC approach of providing integrated services, especially in rural settings, those with low caseloads need access to updated guidelines and mentors to assist them when they initiate patients or encounter complex cases.

\section{Limitations}

Study limitations include the small sample size and high refusal rate, which limits the generalisability of the findings. The small sample size further limits the power of the statistical tests to detect significant differences. As mentioned before, self-assessment may not accurately measure confidence. The cross-sectional nature of the study implies that no cause and effect relationships between the variables explored can be inferred. Further, the knowledge and competency items assessed were delimited to adult HIV care.

\section{Conclusion}

This study investigated various factors that influence the knowledge and confidence of professional nurses prescribing ART in an urban and rural setting, and gave an account of what is currently happening at the healthcare facilities that partook in the study. The majority of participants had adequate HIV management knowledge and reported being very confident or experts in the HIV management skills and competencies. Training, mentorship and clinical practice experience are associated with knowledge and confidence. Recommendations include the strengthening of current training and mentoring programmes and ensuring that NIMART-trained nurses are provided with regular updates and sufficient opportunities for clinical practice.

\section{Acknowledgement Competing interest}

The authors have declared that no competing interests exist.

\section{Author contributions}

D.J.S. wrote the proposal and conducted the study as part of her Master of Nursing degree. T.C. supervised the study and developed the initial draft of the article. A.S.v.d.M. cosupervised the study, and T.M.E. provided statistical support. The article was reviewed by all the authors. 


\section{Funding}

This research received no specific grant from any funding agency in the public, commercial, or not-for-profit sectors.

\section{Data availability statement}

Data sharing is not applicable to this study.

\section{Disclaimer}

The views and opinions expressed in this article are those of the authors and do not necessarily reflect the official policy or position of any affiliated agency of the authors.

\section{References}

1. Mayosi BM, Lawn JE, Van Niekerk A, Bradshaw D, Abdool Karim SS, Coovadia HM. Health in South Africa: Changes and challenges since 2009. Lancet. 2012;380(9858):2029-2043. https://doi.org/10.1016/S0140-6736(12)61814-5

2. Nyasulu JCY, Muchiri E, Mazwi S, Ratshofela M. NIMART rollout to primary health care facilities increases access to antiretrovirals in Johannesburg: An interrupted time series analysis. S Afr Med J. 2013;103(4):232-234. https://doi.org/10.7196/ SAMJ.6380

3. Department of Health (DOH). Global AIDS response progress report. Pretoria: DOH; 2012.

4. Georgeu D, Colvin CJ, Levin S, et al. Implementing nurse-initiated and managed antiretroviral treatment (NIMART) in South Africa: A qualitative process evaluation of the STRETCH trial. Implement Sci. 2012;7:66. https://doi.org/10.1186/1748 5908-7-66

5. World Health Organization (WHO). Task shifting: Rational redistribution of tasks among health workforce teams: Global recommendations and guidelines [homepage on the Internet]. 2008 [cited 2016 May 03]. Available from: http:// www.who.int/healthsystems/TTR-Taskshifting.pdf.

6. International Council of Nurses (ICN). Nursing care continuum-framework and. competencies. Geneva, Switzerland: ICN; 2008.

7. Epstein RM, Hundert EM. Defining and assessing professional competence. J Am Med Assoc. 2002;287(2):226-235. https://doi.org/10.1001/jama.287.2.226

8. Stewart J, O'Halloran C, Barton JR, Singleton SJ, Harrigan P, Spencer J. Clarifying the concepts of confidence and competence to produce appropriate self evaluation measurement scales. Med Educ. 2000;34(11):903-909. https://doi. org/10.1046/j.1365-2923.2000.00728.x
9. Mangi NG. Evaluation of self-efficacy in clinical performance of nurses initiate and management of anti-retroviral therapy by South African professional nurses [homepage on the Internet]. PhD dissertation. University of Fort Hare; 2017 [cited [homepage on the Internet]. PhD dissertation. University of Fort
2018 Jul 24]. Available from: http://hdl.handle.net/10353/4492

10. Rackal JM, Tynan AM, Handford CD, Rzeznikiewiz D, Agha A, Glazier R. Provider training and experience for people living with HIV/AIDS. Cochrane Database Syst Rev. 2011;(6):CD003938. https://doi.org/10.1002/14651858.CD003938.pub2

11. Cameron D, Gerber A, Mbatha M, Mutyabule J, Swart H. Nurse initiation and maintenance of clients on antiretroviral therapy: Are nurses in primary care clinics initiating ART after attending NIMART training? S Afr Med J. 2012;102(2):98-100. https://doi.org/10.7196/SAMJ.5195

12. Mngqibisa R, Muzigaba M, Ncama BP, Pillay S, Nadesan-Reddy N. Upskilling nursing students and nurse practitioners to initiate and manage patients on ART: An outcome evaluation of the UKZN NIMART course. Afr J Health Prof Educ 2017;9(3):153-158. https://doi.org/10.7196/AJHPE.2017.v9i3.879

13. Green A, De Azevedo V, Patten G, Davies MA, Ibeto M, Cox V. Clinical mentorship of nurse initiated antiretroviral therapy in Khayelitsha, South Africa: A quality of care assessment. PLoS One. 2014;9(6):1-6. https://doi.org/10.1371/journal. pone.0098389

14. Jones M, Cameron D. Evaluating 5 years' NIMART mentoring in South Africa's HIV treatment programme: Successes, challenges and future needs. S Afr Med J. 2017;107(10):839-842. https://doi.org/10.7196/SAMJ.2017.v107i10.12392

15. Visser CA, Wolvaardt JE, Cameron D, Marincowitz GJO. Clinical mentoring to improve quality of care provided at three NIM-ART facilities: A mixed methods study. Afr Prim Health Care Fam Med. 2018;10(1):a1579. https://doi.org/10.4102/ phcfm.v10i1.1579

16. Orner $P$, Cooper $D$, Palmer N. Investigation of health care workers' responses.to HIV/AIDS care and treatment in South Africa [thesis]. Woman's Health Research Unit, School of Public Health and Family Medicine, Faculty of Health Sciences, University of Cape Town, South Africa; 2010.

17. Morris MB, Chapula BC, Chi BH, et al. Use of task-shifting to rapidly scale-up HIV treatment services: Experiences from Lusaka, Zambia. BMC Health Serv Res. 2009;9(5):1-9. https://doi.org/10.1186/1472-6963-9-5

18. Smith J, Odera DN, Chege D, et al. Identifying the gaps: An assessment of nurses' training, competency, and practice in HIV care and treatment in Kenya. J Assoc Nurs AIDS Care. 2016;27(3):322-330. https://doi.org/10.1016/j.jana.2016. 01.005

19. Burns N, Grove SK. Understanding nursing research. Building an evidence-based practice. 5th ed. London: Elsevier; 2011.

20. Polit DF, Beck CT. Essentials of nursing research appraising evidence for nursing practice. 8th ed. Philadelphia, PA: Lippincott Williams \& Wilkins; 2014

21. Crowley T. The development of an instrument to evaluate the HIV/TB management confidence and knowledge of nurses. Poster presentation at the South African HIV Clinicians Society Conference; 2014 Sep. p. 58.

22. IBM Corp. IBM SPSS Statistics for Windows. Version 24.0. [Computer software]. Armonk, NY: IBM Corp; 2016.

23. Department of Health (DOH). Clinical mentorship manual for integrated services. Pretoria: DOH; 2011. 